\title{
Analysis and application of prediction model for formation fluid sampling time while drilling
}

\author{
Zhongshuai Chen ${ }^{1}$, Hongjian Ni, ${ }^{1,}$, Chuanlong Jiang ${ }^{2}$, Yang Zhang ${ }^{2}$ and Hui Zhang ${ }^{3}$ \\ ${ }^{1}$ School of Petroleum Engineering, China University of Petroleum (East China), Qingdao 266580, Shandong,China; \\ 2. College of Mechanical and Electrical Engineering, Beijing University of Chemical Technology ,Beijing ,China; \\ ${ }^{3 .}$ Drilling Technology Research Institute of Shengli Oilfield Service Corporation ,Sinopec ,Dongying, Shandong,China;
}

\begin{abstract}
Formation sampling while drilling (FSWD) is one of the most advanced formation sampling techniques in the world. It is characterized by short operation time, shallow mud invasion, and the obtained formation data is closer to the real situation of the reservoir. During the sampling process, the first fluid that the suction probe on the borehole wall inhales is basically drilling mud filtrate. With the increase of suction time, it gradually mixes into the formation fluid, and finally the formation fluid is infinitely close to $100 \%$. At this time, it is very important to judge the starting time of sampling. If the sampling time is too early, the percentage of samples contaminated by drilling filtrate will be higher, which can not meet the requirement of fidelity. If the sampling time is too late, the drilling fluid will stop circulating for too long, which will cause sticking. In this paper, the prediction method of sampling time is to simulate the permeability of drilling fluid to formation under certain formation parameters and drilling conditions. Based on the simulated permeability results, the suction model of sampling tool is established to simulate the pumping situation, and the variation of the content of drilling filtrate in the pumped fluid with the suction time is obtained, that is, the relationship between the contamination rate of formation fluid and the suction time, This method is of great significance to the field application of fluid sampling tools while drilling.
\end{abstract}

\section{Introduction}

It is very important to obtain geological parameters such as fluid type and property by collecting representative formation fluid samples, which is of great significance to accurately determine the oil-gas situation of reservoir and reasonably formulate medium and long-term development plan of oil and gas field ${ }^{[1-2]}$. At present, there are two main ways to obtain formation fluid: wireline formation sampling and formation sampling while drilling (FSWD) .

Wireline formation sampling technology has been developed for a long time and the technology is mature now. However, in the process of sampling, the instrument is easy to stick because the drilling fluid stops circulating for too long. Moreover, due to the sampling after drilling, the formation is opened for a long time, and the formation near the wellbore is seriously polluted by mud filtrate, so it is difficult to obtain the real composition data of formation fluid ${ }^{[3]}$. FSWD is developed on the basis of wireline formation sampling, formation pressure testing while drilling, and formation fluid analysis technology. It can quickly collect low pollution or non pollution formation fluid when the oil and gas reservoir is just opened. The operation time is short, the mud invasion is shallow, and the formation data obtained are closer to the real situation of the formation ${ }^{[4-6]}$.
In the formation of fluid sampling while drilling, foreign research has a history of more than 20 years. In recent years, Schlumberger, Halliburton and Baker Hughes have launched their own characteristic products ${ }^{[7-}$ ${ }^{8]}$. Among them, there are many literatures about mud infiltration or sampling and pumping formation fluid. Zazovsky(2008) proposed a model which is a combination of practical experience and simulation calculation. Its fundamental basis is to monitor the fluid composition sucked by downhole probe through optical fluid analyzer (OFA), analyze the optical density difference between formation fluid and drilling filtrate invading formation, reflect the progress of formation fluid displacement drilling filtrate through optical density change, and judge the beginning of sampling time ${ }^{[9-13]}$.

In China, CNOOC oilfield services [14-15] and PetroChina Daqing drilling engineering company ${ }^{[16]}$ have made corresponding attempts in pressure and temperature measurement while drilling, but generally speaking, they are still in the stage of importing and imitating corresponding foreign instruments, and there are still some deficiencies in technical level, technical indicators and field application, especially in the absence of downhole opto-fluid analyzers, sampling time prediction is blank.

*Correspondence should be addressed to Hongjian Ni; nihj@upc.edu.cn. 


\section{Design and research of related parameters}

In the process of drilling, the water column pressure of drilling fluid is slightly higher than that of the original formation, which leads to the penetration of drilling fluid into the original formation. The part of drilling fluid that intrudes into the formation is called drilling filtrate. Generally speaking, according to the saturation of the invasion into the formation, it can be divided into three areas: flushed zone, transition zone and original formation, as shown in Figure1. In fact, the radius and saturation of the invasion of drilling filtrate are gradually changing with the invasion time, pressure difference, filtrate viscosity, reservoir permeability and cake permeability. When sampling while drilling under such permeation conditions, the content of drilling filtrate in the pumped formation fluid, that is, when the contamination rate of the pumped formation fluid reaches the sampling requirement, also varies with the filtrate infiltration time, pressure difference, filtrate viscosity, reservoir permeability, pumping capacity and cake permeability.

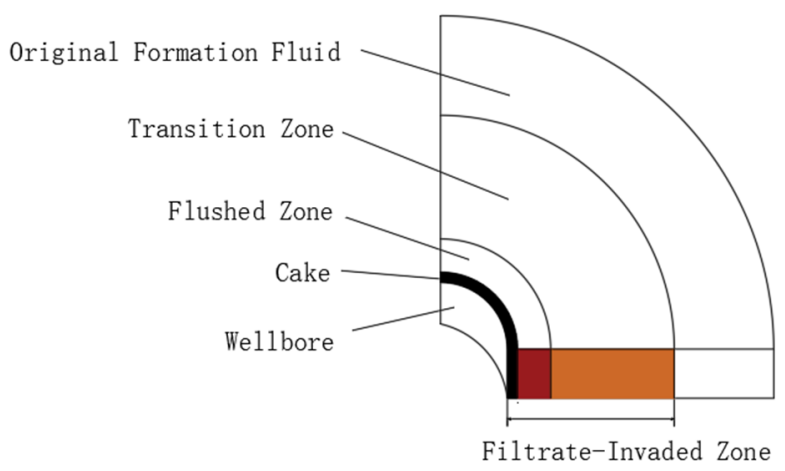

Figure 1. Profile of drilling filtrate invasion zone

According to the data and analysis, the formation property and fluid property parameters of an oil area in Shengli are as follows ${ }^{[17]}$ :

1) For medium and high permeability reservoir $\left(K_{1}>\right.$ $50 \mathrm{mD}$ ), the average formation permeability is $89.6 \mathrm{mD}$, and the porosity of this kind of reservoir $\Phi_{1}$ is $21.9 \%$. For general low permeability reservoir $\left(10.2 \mathrm{mD}<\mathrm{K}_{1}<\right.$ $42.3 \mathrm{mD}$ ), the average formation permeability is $12.5 \mathrm{mD}$, and the porosity of this kind of reservoir $\Phi_{1}$ is $12.4 \%$.

2)Generally, the permeability of mud cake is $1-3$ orders of magnitude smaller than that of formation. Assuming that mud cake is well formed, in medium and high permeability reservoirs, the formation permeability is three orders of magnitude of mud cake permeability; in general low permeability reservoirs, the formation permeability is two orders of magnitude of mud cake permeability, and the unified porosity of mud cake $\Phi_{2}$ is $3 \%$.

3) The over balanced pressure difference range of drilling fluid during drilling cycle $\mathrm{P}$ is $1-4 \mathrm{MPa}$.

4) The viscosity of drilling fluid $\mu_{1}$ is $25-35 \mathrm{mPa} \cdot \mathrm{s}$.

5) The viscosity range of crude oil $\mu_{2}$ is $25-100 \mathrm{mPa} \cdot \mathrm{s}$.

6) The unified drilling fluid density $\rho_{1}$ is $1100 \mathrm{~kg} / \mathrm{m}^{3}$ and the crude oil density $\rho_{2}$ is $850 \mathrm{~kg} / \mathrm{m}^{3}$.
During the analysis and calculation, the formation permeability $\mathrm{K}_{1}$ is divided into 8 grades: $90 \mathrm{mD}, 75 \mathrm{mD}$, $60 \mathrm{mD}, 45 \mathrm{mD}, 40 \mathrm{mD}, 30 \mathrm{mD}, 20 \mathrm{mD}$ and $10 \mathrm{mD}$, the overbalance pressure difference $\mathrm{P}$ is divided into 4 grades: $1 \mathrm{MPa}, 2 \mathrm{MPa}, 3 \mathrm{Mpa}$ and $4 \mathrm{MPa}$, the crude oil viscosity $\mu_{2}$ is divided into 4 grades: $25 \mathrm{mPa} \cdot \mathrm{s}, 50 \mathrm{mPa} \cdot \mathrm{s}, 75 \mathrm{mPa} \cdot \mathrm{s}$ and $100 \mathrm{mPa} \cdot \mathrm{s}$, the invasion time $t$ is divided into 6 grades:30min, 60min, 90min, 120min, 150min and $180 \mathrm{~min}$, the viscosity of drilling fluid $\mu_{1}$ is selected as 30 $\mathrm{mPa} \cdot \mathrm{s}$ (In discussion 2, the simulation shows that the viscosity of drilling fluid changes in the range of 25-35 $\mathrm{mPa} \cdot \mathrm{s}$, which has little effect on the sampling time and can be ignored), and other parameters remain unchanged. Among them, a total of 768 different working conditions were established through the combination of different parameters and multi working conditions, and the calculation models of filtrate invasion into the formation and fluid sampling time were established. Through the results of these examples, a data table which can envelope and radiate to various geological conditions and drilling conditions is summarized to determine the sampling time, so as to guide the actual operation of sampling time.

\section{Simulation of infiltration process}

In order to simplify the modeling and analysis of drilling fluid invasion process, the following assumptions are made: there is oil-water two-phase flow in the reservoir, all fluids in the reservoir conform to Darcy's law and mass conservation equation., the compressibility of crude oil and filtrate is ignored, the density, temperature and viscosity of formation fluid are constant, the rock in the reservoir is isotropic and homogeneous, all the flow in the process of drilling fluid invasion is radial flow.

The seepage between oil-water phases in reservoirs is not considered by capillary force, and the seepage satisfies the following equation.

The equation of motion of oil-water two-phase seepage:

Oil phase:

$$
\overline{v_{o}}=-\frac{K_{o}(s)}{\mu_{o}} \operatorname{gradP}
$$

Water phase:

$$
\overline{v_{w}}=-\frac{K_{w}(s)}{\mu_{o}} \operatorname{gradP}
$$

The continuity equation of oil-water two-phase flow is as follows:

Oil phase:

$$
-\left(\frac{\partial v_{o x}}{\partial x}+\frac{\partial v_{o y}}{\partial y}+\frac{\partial v_{o z}}{\partial z}\right)=\varnothing \frac{\partial S_{o}}{\partial t}
$$

Water phase:

$$
-\left(\frac{\partial v_{w x}}{\partial x}+\frac{\partial v_{w y}}{\partial y}+\frac{\partial v_{w z}}{\partial z}\right)=\emptyset \frac{\partial S_{w}}{\partial t}
$$

If there is no mass transfer between oil and filtrate, and the pore space is filled together, the oil saturation $S_{o}$ and filtrate saturation $S_{w}$ are as follows:

$$
S_{o}+S_{w}=1
$$


Where:

$K_{o}(s), K_{W}(s)$ are the phase permeability of oil and water respectively.

$S_{o}, S_{w}$ are oil phase saturation and filtrate saturation respectively.

The phase conduction of porous media in the fluid flow module of Darcy Two-phase flow are used to solve the simulation. Two phase flow is also used to simulate the process of fluid invasion by drilling filtrate.

A two-dimensional geometric model is established in the plane perpendicular to the drill pipe axis at the sampling formation position. Due to the geometric symmetry of the model, a quarter circle is established to simulate the formation for solution. The geometric model is shown in Figure 2. Its radius is half of the wellbore diameter of $215.9 \mathrm{~mm}$, the reservoir radius is $5 \mathrm{~m}$, and the thickness of mud cake is $2 \mathrm{~mm}$. Set the boundary conditions, boundary 1 and 2 can not flow, boundary 3 is set at the inlet, boundary 4 is set at the outlet, the saturation of filtrate at the wellhead boundary is set as $S_{1}=1$, and the saturation of remaining oil is set as $\mathrm{S}_{2}=0$.

The structural grid is used for the grid generation, and the transient solution is carried out to find out the law of intrusion radius changing with time under a certain saturation.

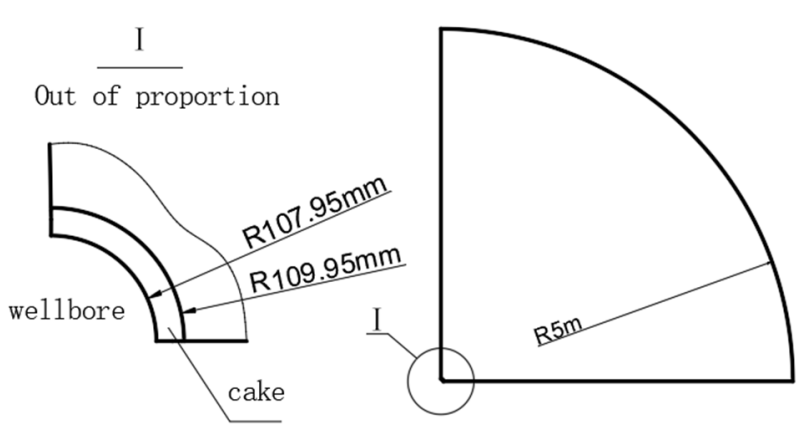

(a) The geometric model

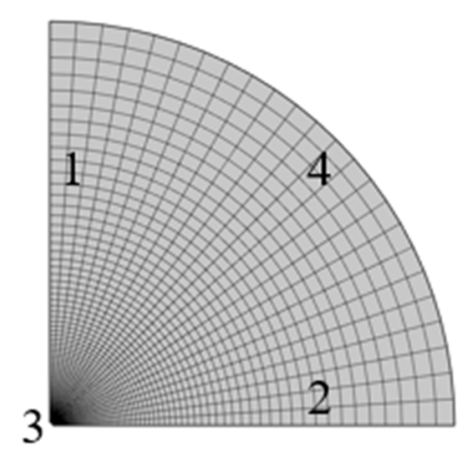

(b) Meshing model

Figure 2. Reservoir geometry model and grid generation

Example 1: The overbalance pressure difference $\mathrm{P}$ is $3 \mathrm{MPa}$, the formation permeability $\mathrm{K}_{1}$ is $45 \mathrm{mD}$, which belongs to the medium high permeability reservoir,the formation porosity $\Phi_{1}$ is $21.9 \%$, the drilling fluid viscosity $\mu_{1}$ is $30 \mathrm{mPa} \cdot \mathrm{s}$, and the crude oil viscosity $\mu_{2}$ is $25 \mathrm{mPa} \cdot \mathrm{s}$. according to the above 2 (2), the medium high permeability formation permeability is three orders of magnitude of the mud cake permeability, so the mud cake permeability $\mathrm{K}_{2}$ is $0.045 \mathrm{mD}$, the porosity of mud cake $\Phi_{2}$ is $3 \%$, the drilling fluid density $\rho_{1}$ is $1100 \mathrm{~kg} / \mathrm{m}^{3}$, the crude oil density $\rho_{2}$ is $850 \mathrm{~kg} / \mathrm{m}^{3}$, and the invasion time $t$ is $120 \mathrm{~min}$. The parameter in example 1 is one of the above 768 working conditions, and the permeability nephogram is shown in Figure 3. The color of radial saturation changes from red to blue with the increase of permeability radius, and the change gradient is completely consistent in circumferential direction, which means that the saturation of drilling fluid changes from $100 \%$ to 0 with the increase of permeability radius in formation outside wellbore. In this example, the curve of permeability time corresponding to formation saturation changing with permeability radius is shown in Figure 4.

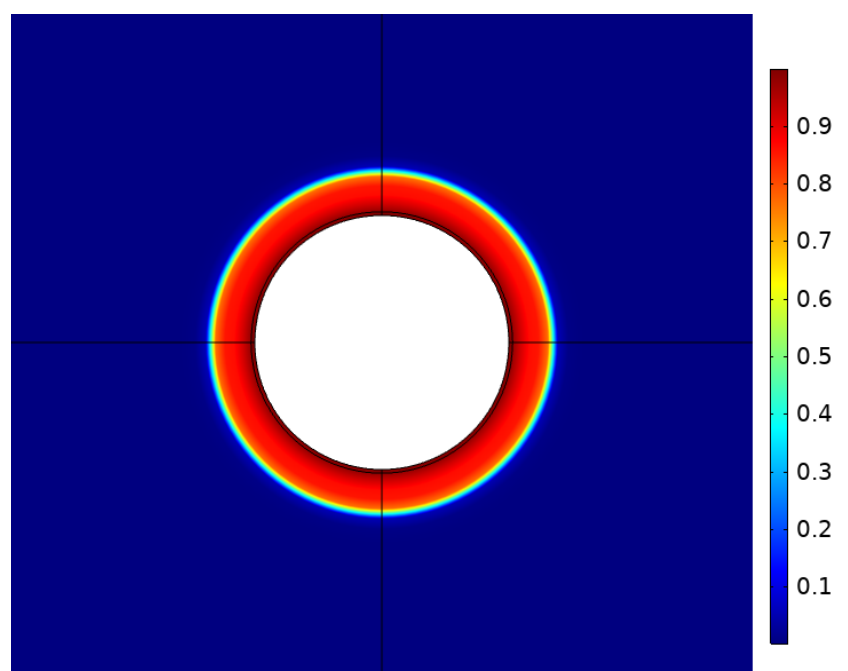

Figure 3. Example 1 cloud image of formation permeability for $120 \mathrm{~min}$ 


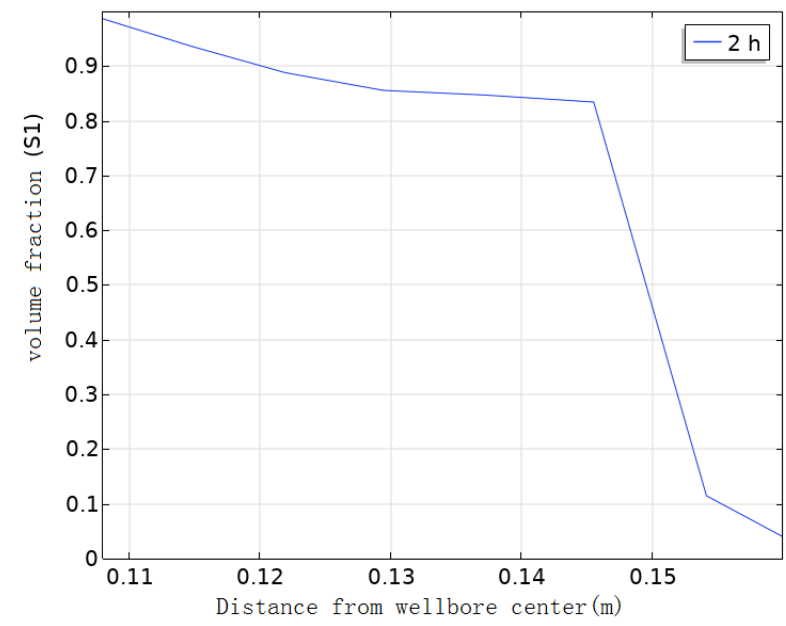

Figure 4. Variation of formation saturation with permeability radius after $120 \mathrm{~min}$ permeability

\section{Simulation of suction process}

The suction function of the sampling tool is to press a

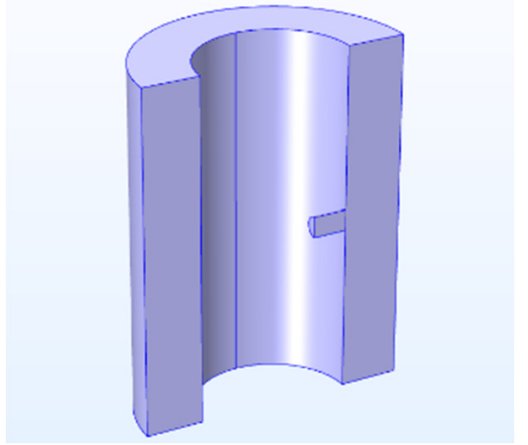

(a) The geometric model miniature probe with cushion on the well wall, then reduce the pressure in the probe to enough to destroy the mud cake near the probe and extract the fluid from the formation.

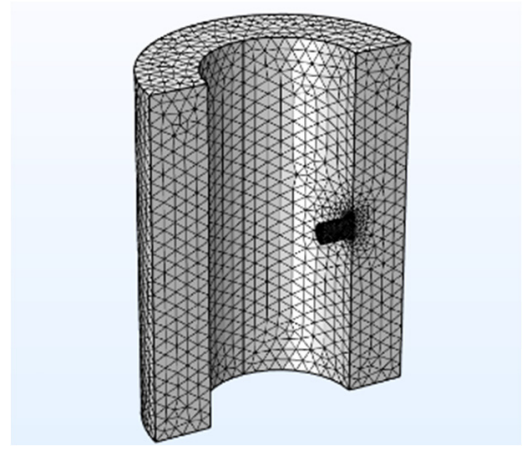

(b) Meshing model

Figure 5. Suction process calculation model

During the simulation, a three-dimensional hollow cylinder is used to simulate the formation. The axis of the cylinder is in the same direction as the axis of the drill pipe. Due to the geometric symmetry of the model, a half cylinder is established to simulate the suction process. The inner diameter of the cylinder is $215.9 \mathrm{~mm}$, and the outer diameter is $290 \mathrm{~mm}$. The wall thickness is $37 \mathrm{~mm}$, which is the maximum thickness of $1 \mathrm{~h}$ invasion in example 1 . The height of the cylinder is $500 \mathrm{~mm}$. A tube with an inner diameter of $30 \mathrm{~mm}$ is set in the middle of the cylinder to simulate the suction probe, as shown in Figure 5(a), Figure 5 (b) shows its meshing model. This model is based on the fact that the pressure in the probe is reduced enough to destroy the mud cake near the probe, so the mud cake modeling can be ignored.

Example 2. Input the parameters of the corresponding reservoir infiltration model in example 1 , and import the relationship curve between the saturation and the radius of the wellbore in example 1 to define the saturation of the filtrate in the reservoir, which is known as the known pollution situation of the stratum as shown in Figure 6. At this point, the radial color gradient is consistent.

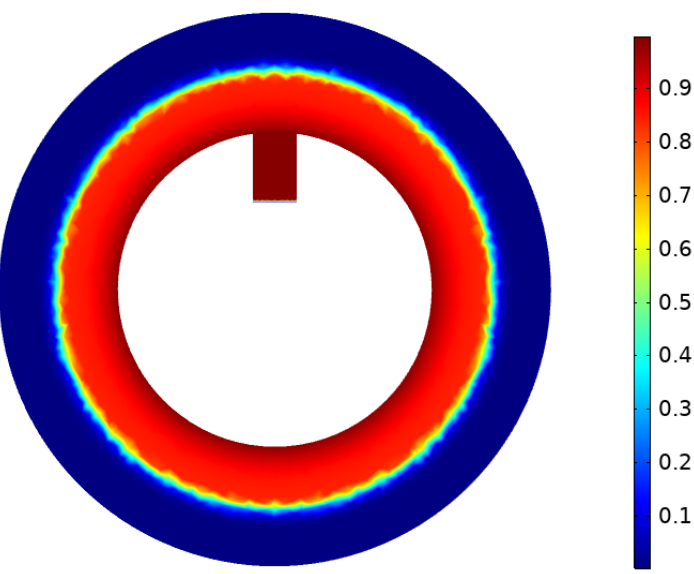

(a) Circumferential flow field of reservoir 

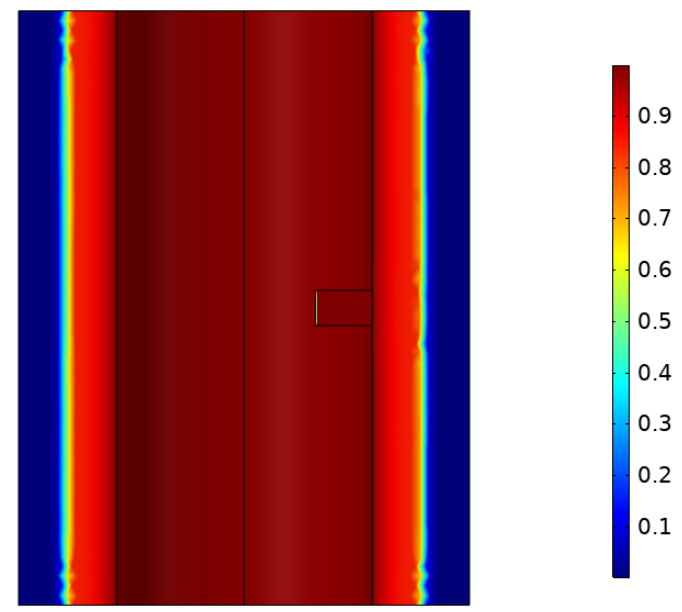

(b) Axial flow field of reservoir

Figure 6. In example 2, the saturation distribution of reservoir drilling filtrate at $0 \mathrm{~min}$ suction process

In the case of unknown formation fluid bubble point, in order to make the outlet pressure of the suction probe and the formation pressure of the sample have little difference, the suction pressure difference should be maintained at $3 \mathrm{MPa}$, so as to prevent the phase transformation of the sample during the suction process. Figure 7 shows the saturation distribution of the drilling filtrate in the reservoir model at $121 \mathrm{~min}$ during the pumping process of example 2, which shows that the radial color gradient is no longer consistent. During the pumping process, a cone-shaped flow field distribution is formed at the entrance of the probe, and the formation fluid flows towards the probe head. With the continuous pumping, the flow field velocity near the pipe wall of the probe suction inlet decreases Because of the difference between the direction of suction and that of suction, the degree of contamination is always higher than that of the area with the same direction of suction, which leads to the contamination rate at the wall of the probe always higher than that at the center of the probe. Figure 8 shows the curve of the average pollution rate of the filtrate at a certain cross section of the probe with time. It shows that with the increase of the suction time, the pollution rate of the drilling filtrate rapidly decreases from $100 \%$ to $15 \%$ within 20 minutes of the sampling time. After 20 minutes, the pollution rate decreases slowly and finally infinitely approaches but is not equal to $0 \%$. When the sampling time is 121 minutes, the pollution rate is $5 \%$, and the qualified samples can be obtained (the pollution rate of drilling filtrate in the design fluid sample is less than 5\%).

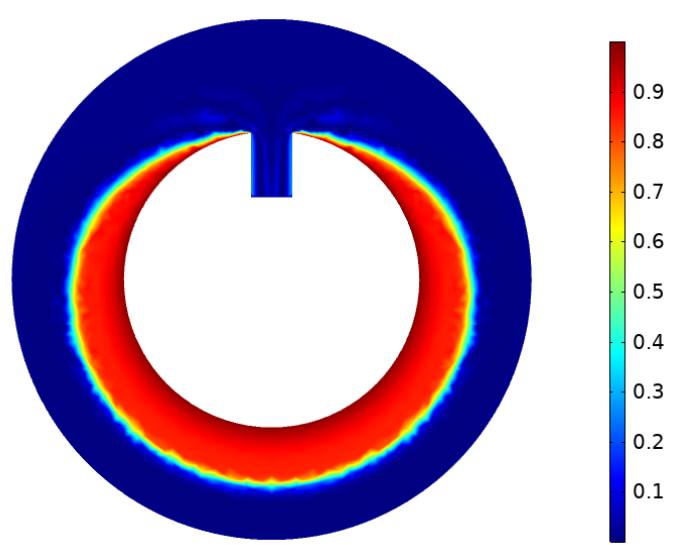

(a) Circumferential flow field of reservoir

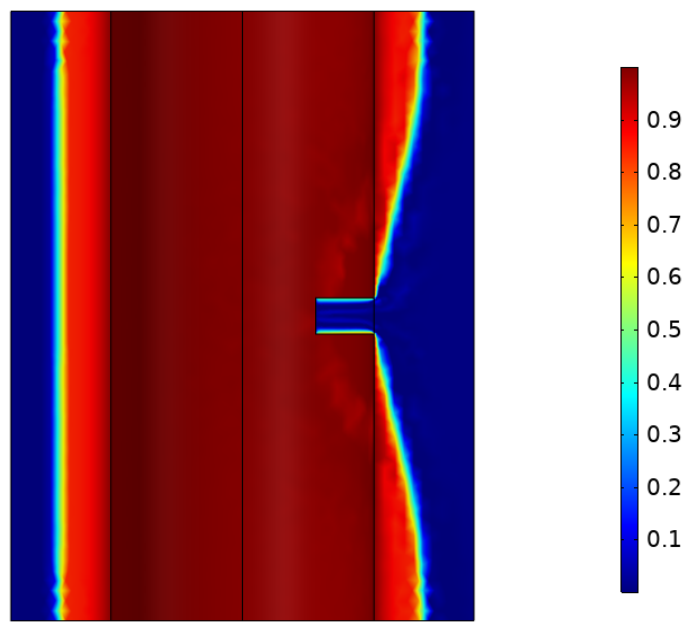

(b) Axial flow field of reservoir

Figure 7. In example 2, the saturation distribution of reservoir drilling filtrate at $121 \mathrm{~min}$ suction process

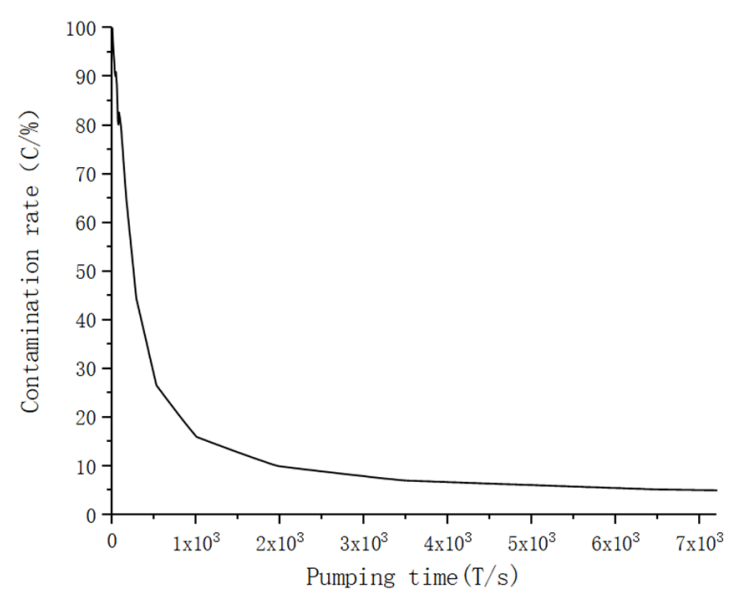

Figure 8. In example 2, the variation curve of the percentage of drilling filtrate (contamination rate) in the fluid pumped by the probe with time

\section{List and application of each example}

The relevant condition parameters and simulation results of example 1 and example 2 are listed in the parameter group number 400 in Table 1 in combination order. According to the seepage simulation calculation method in case 1 and the suction simulation calculation method in example 2, the seepage and simulation calculation of 768 
working conditions mentioned in example 1 are completed, and the results are summarized in Table 1, as shown in Table 1. In practical application, two methods can be adopted.

(1) If the relevant parameters of the sampling formation and drilling are exactly consistent with the gear in the serial number of a parameter group in Table 1, the sampling time can be directly checked for reference, if it is very close to a parameter gear in Table 1 , about $20 \%$ above and below the gear value, it can be similar to that gear to look up the table. For example, when $\mathrm{K}_{1}=47 \mathrm{mD}, \mu_{2}=26$ $\mathrm{mPa} \cdot \mathrm{s}, \mathrm{P}=2.8 \mathrm{MPa}, \mathrm{t}=120 \mathrm{~min}$, It can be approximated to $\mathrm{K}_{1}=45 \mathrm{mD}, \mu_{2}=25 \mathrm{mPa} \cdot \mathrm{s}, \mathrm{P}=3 \mathrm{MPa}, \mathrm{t}=120 \mathrm{~min}$, that is, 400 serial number parameter group in Table 1, then sampling time of $121 \mathrm{~min}$ can be obtained directly. Compared with the actual simulation calculation, the error is $+16 \%$. Under the condition of sacrificing sampling time, the pollution rate of the retrieved sample can be less than $5 \%$, which can meet the sampling requirements.

(2) If the relevant parameters of sampling formation and drilling are between two working condition parameter serial numbers, and deviate more than $20 \%$ from the gear in the serial number of a parameter group in Table 1 , the average conservative method can be used to determine the sampling time. For example, when $\mathrm{K}_{1}=56 \mathrm{mD}, \mu_{2}=32$ $\mathrm{mPa} \cdot \mathrm{s}, \mathrm{P}=2.8 \mathrm{MPa}, \mathrm{t}=114 \mathrm{~min}$, The overbalance pressure difference is within the range of $2-3 \mathrm{MPa}$ in Table 1 ,so the larger value of $\mathrm{P}$ is $3 \mathrm{Mpa}$, and the invasion time is within the range of $90-120 \mathrm{~min}$ in Table 1 , so the value of $t$ is $120 \mathrm{~min}$, The formation permeability and viscosity of crude oil can be discussed as follows: (1) $\mathrm{K}_{1}=45 \mathrm{md}$, $\mu_{2}=25 \mathrm{mPa} \cdot \mathrm{s}, \mathrm{P}=3 \mathrm{MPa}, \mathrm{t}=120 \mathrm{~min}$; (2) $\mathrm{K}_{1}=45 \mathrm{md}$, $\mu_{2}=50 \mathrm{mPa} \cdot \mathrm{s}, \mathrm{P}=3 \mathrm{MPa}, \mathrm{t}=120 \mathrm{~min}$ (3) $\mathrm{K}_{1}=60 \mathrm{md}$, $\mu_{2}=25 \mathrm{mPa} \cdot \mathrm{s}, \mathrm{P}=3 \mathrm{MPa}, \mathrm{t}=120 \mathrm{~min}$ (4) $\mathrm{K}_{1}=60 \mathrm{md}$, $\mu_{2}=50 \mathrm{mPa} \cdot \mathrm{s}, \mathrm{P}=3 \mathrm{MPa}, \mathrm{t}=120 \mathrm{~min}$, corresponding to 400 , 424, 496 and 520 condition numbers respectively. The average sampling time of the four groups is $119.5 \mathrm{~min}$, and the error is $+25 \%$ compared with the actual simulation value. Under the condition of sacrificing sampling time, the pollution rate of the retrieved samples can be less than $5 \%$, which can also meet the sampling requirements.

Table1. List of sampling time required under different reservoir and drilling parameters under suction pressure drop of $3 \mathrm{MPa}(\mathrm{Partial}$ display)

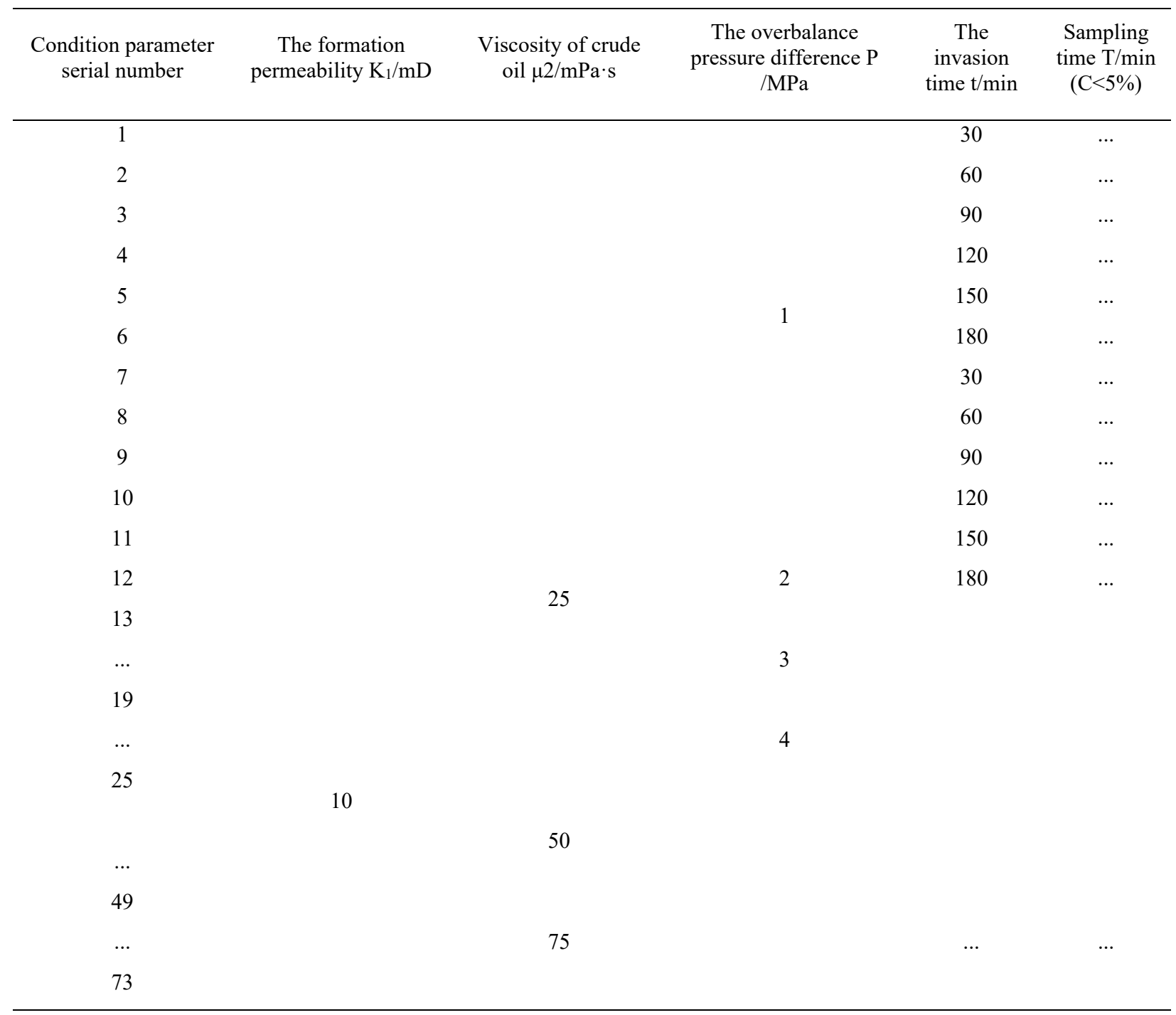




\begin{tabular}{|c|c|c|c|c|c|}
\hline$\ldots$ & & 100 & $\ldots$ & & \\
\hline \multicolumn{6}{|l|}{97} \\
\hline$\ldots$ & 20 & & & & \\
\hline \multicolumn{6}{|l|}{193} \\
\hline$\ldots$ & 30 & & & & \\
\hline 289 & & $\ldots$ & & & \\
\hline$\ldots$ & 40 & & & & \\
\hline \multicolumn{6}{|l|}{385} \\
\hline \multicolumn{6}{|l|}{$\ldots$} \\
\hline 400 & & 25 & 3 & 120 & 121 \\
\hline \multicolumn{6}{|l|}{$\ldots$} \\
\hline \multicolumn{6}{|l|}{409} \\
\hline \multicolumn{6}{|l|}{$\ldots$} \\
\hline 424 & & 50 & 3 & 120 & 88 \\
\hline$\ldots$ & 45 & 0 & & & \\
\hline$\ldots$ & & $\ldots$ & & & \\
\hline 481 & & & $\ldots$ & $\ldots$ & $\ldots$ \\
\hline \multicolumn{6}{|l|}{$\ldots$} \\
\hline 496 & & 25 & 3 & 120 & 162 \\
\hline \multicolumn{6}{|l|}{$\ldots$} \\
\hline \multicolumn{6}{|l|}{504} \\
\hline$\cdots$ & & 50 & & & \\
\hline 520 & & & 3 & 120 & 107 \\
\hline$\ldots$ & 60 & & & & \\
\hline \multicolumn{6}{|l|}{$\ldots$} \\
\hline \multicolumn{6}{|l|}{577} \\
\hline$\ldots$ & 75 & & $\ldots$ & $\ldots$ & \\
\hline 673 & & $\ldots$ & & & $\ldots$ \\
\hline \multicolumn{6}{|l|}{$\ldots$} \\
\hline 768 & 90 & 100 & 4 & 180 & \\
\hline
\end{tabular}

\section{Discussion}

(1) The purpose of this paper is to explain how to predict the sampling time by simulation. The gear setting and density of formation permeability and crude oil viscosity in Table 1 are determined according to the situation of an oil area in Shengli. If other oil areas have relevant parameters falling in the middle of the two gears in Table 1 , there will be some errors in determining the sampling time by method 2, and the larger the gear spacing is, the greater the error is .Therefore, in order to reduce the error, the corresponding parameters in Table 1 can be divided into more detail, and more combined examples can be added, so that more accurate sampling time can be obtained, and more practical application of field conditions can be convenient.
(2) In the simulation, if the viscosity of drilling fluid $\mu_{1}$ is $25-35 \mathrm{mPa} \cdot \mathrm{s}$, it can be divided into three grades $25 \mathrm{mPa} \cdot \mathrm{s}, 30 \mathrm{mPa} \cdot \mathrm{s}$ and $35 \mathrm{mPa} \cdot \mathrm{s}$. The other parameters are the same as those in example 1 to simulate the permeability process. Then, based on the permeability results, the suction process is simulated according to example 2. The relationship between the pollution rate and the corresponding suction time is shown in Figure 9. It can be seen that when the viscosity of drilling fluid changes in a small range, it has little effect on the final sampling time. Therefore, the viscosity of drilling fluid in Table 1 is calculated by taking the middle value of $30 \mathrm{mPa} \cdot \mathrm{s}$. If the variation range of drilling fluid viscosity is larger than $30 \mathrm{MPa} \cdot \mathrm{s}$, it is necessary to add the range of drilling fluid viscosity in Table 1, and the number of parameter groups and the amount of simulation calculation are doubled. 


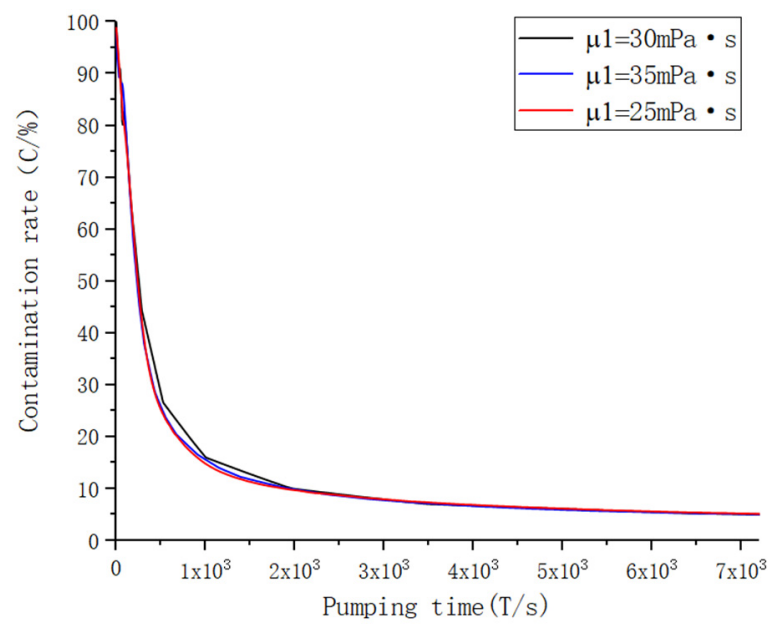

Figure 9. The variation curve of the percentage of drilling filtrate (pollution rate) in the fluid pumped by the probe with time under different drilling fluid viscosities

(3) The sampling time listed in Table 1 is based on the suction pressure drop of $3 \mathrm{MPa}$. If it is known that the sampling formation pressure is far greater than the bubble point pressure of the formation fluid, the suction pressure drop can be increased, and the sampling time will be shortened as a result. In this way, the data in Table 1 needs to be replaced with a large suction pressure drop, and the same simulation method is needed to recalculate.

\section{References}

1. M.Meister, J.Pragt, and A.Buysch, "Pressure gradient testing with a new formation pressure testing during drilling tool,"SPE 90425, 2004.

2. S.Villareal, J.Pop, and F.Bernard, "Characterization of sampling while drilling operations," SPE128249,2010.

3. G.Q.Zhu, Z.Q.Zhang, "New development of wireline logging technology abroad, Logging technology,"vol.32,no.6,pp.380-384,2008.

4. F.Galvan Sanchez, A.Cartellieri, J.Pragt, and M.Meister, "Fluid Analysis and Sampling:The Next Big Step for Logging While Drilling Tools," Latin America and Caribbean Petroleum Engineering Conference,SPE 152223 ,DOI:10.2118/152223ms,2012.

5. M.A.Proett, S.Eyuboglu, J.R.Wilson, and T.H.N.Solbakk, "New Sampling and Testing-WhileDrilling Technology - A Safe, Cost-Effective Alternative," SPE/IADC Drilling Conference and Exhibition,DOI:10.2118/140337-ms,2011.

6. M.Djefel, C.Tigre, M.Proett, "Potential economic value-added model using formation sampling-whiledrilling in development fields," SPE 138956,2010.

7. A.Zazovsky, "Monitoring and Prediction of Cleanup Production During Sampling, "Presented at the SPE International Symposium and Exhibition on Formation Damage Control , Lafayette , Louisiana, USA, SPE-112409-MS , 13-15
February, 2008.

8. A.H.Akram, A.J.Fitzpatrick, and F.R.Halford, "A Model to Predict Wireline Formation Tester Sample Contamination,"SPEREE vol.2,nol.6, pp.499-505. SPE-59559-PA, 1999.

9. P.S.Hammond, "One- and Two-Phase Flow During Fluid Sampling by a Wireline Tool, "Transport in Porous Media vol,6.pp. 299-330,1991.

10. F.O.Alpak, H.Elshahawi, M.Hashem, and O.Mullins, "Compositional Modeling of Oil-Based Mud-Filtrate Cleanup During WirelineFormation Tester Sampling,"Paper SPE 100393 presented at the 2006 SPE Annual Technical Conference and Exhibition, SanAntonio, Texas, 24-27 September,2006.

11. O.C.Mullins, and J.Schroer, "Real-Time Determination of Filtrate Contamination during Openhole Wireline Sampling by Optical Spectroscopy,"Paper SPE 63071 presented at the 2000 SPE Annual Technical Conference and Exhibition, Dallas, Texas, 1-4 October,2000.

12. S.Prensky, "Recent Development in well Logging and Formationg Evaluation," World oil , vol.233,no.6,pp.79-84,2012.

13. M.G.Zhou, S.M.LIU, and Y.R.Feng, "Development and application of FCT during drilling," Logging technology, vol.32,no.1,pp.72-75,2008.

14. Q.Yu,S.G.Liu,and S.M.Liu,"Reservoir tester (ERCT) and its application in Bohai oilfield," Petroleum instruments, vol.25,no.6,pp.23-25,2011.

15. Z.X.Zhao, Y.A.Han, and X.Gao,"SDC-I formation pressure tester while drilling," Petroleum machinery, vol.39,no.2,pp.52-54,2011.

16. K.X.Hu, X.H.Wang, "Study on the relationship between porosity and permeability of various reservoirs," Petrochemical applications, vol.33,no.11, pp.40-42,2014.

17. Y.R,Fan, Z.G.Wu, F.Wu, J.C.Wu, and L.Wang. "Formation module scale drilling fluid invasion simulation and reservoir resistivity profile characteristics," Petroleum exploration and development, vol.44,no.6,pp.989-996,2017. 